\title{
Quantitative Analysis of Nucleosides and Nucleobases in Deer Antler: Variation in Different Species
}

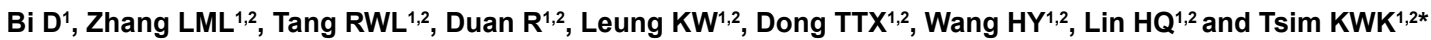 \\ ${ }^{1}$ Division of Life Science and Center for Chinese Medicine, The Hong Kong University of Science and Technology, Clear Water Bay Road, Hong Kong, China \\ ${ }^{2}$ HKUST Shenzhen Research Institute, Hi-Tech Park, Nanshan, Shenzhen 518000, Guangdong Province, China
}

\begin{abstract}
An HPLC-diode array detector analytical method was developed for the determination of 10 nucleosides and nucleobases, e.g. uracil, cytidine, uridine, hypoxanthine, inosine, guanine, guanosine, thymidine, adenosine and adenine, in 29 batches of deer antlers deriving from 11 species of deer: the official species of Cervi Cornu Pantotrichum recorded in Chinese Pharmacopoeia, i.e., Cervus nippon Temminck and Cervus elaphus Linnaeus, were included. This established HPLC-diode array detector method was validated to be sensitive, precise and accurate in determining the nucleosides and nucleobases of deer antlers. Quantitative analyses showed that most of deer antlers contained high amounts of nucleosides and nucleobases, except the amounts of cytidine, thymidine, adenosine and adenine; however, which varied greatly in different species and different regions of collection. The similarity of chemical fingerprints was determined, and the antlers from C. nippon and C. elaphus showed the highest similarity, suggesting the possible application in authentication. However, the species discrimination of deer antler could not be fully revealed by hierarchical cluster analysis (HCA) and/or principal component analysis (PCA).
\end{abstract}

Keywords: Deer antler; Nucleosides; Nucleobases; Quantitative analysis; HPLC-diode array detector

\section{Introduction}

Deer antler, Cervi Cornu Pantotrichum or called "Lurong" in China, is an valuable traditional Chinese medicine (TCM), recorded in the Compendium of Materia Medica (Bencao Gangmu) by Li ShiZhen in 1594 AD. Deer belongs to a family Cervidae having 10 genus and 17 species in China. Most of these species are commonly used in folk medicine in reinforcing kidney-yang, replenishing vital essence and blood, and strengthening the tendons and bones for thousands of years $[1,2]$. Additionally, deer antler has been widely sold as a dietary supplement in various Asian countries such as in China and Korea. According to Chinese Pharmacopoeia, the official source of Cervi Cornu Pantotrichum should be from the antlers of Cervus nippon Temminck and Cervus elaphus Linnaeus only [1]. In view of its expensive cost, the counterfeits of antler are commonly found in the market.

Deer antler is frequently consumed as a health food supplement in China. Pharmacological studies indicated that deer antler could be used to delay aging-related processes, to improve sexual function, to modulate immune responses and to lower blood pressure [3-5]. Deer antler contains various chemical compositions, including protein, amino acid, phospholipid, polysaccharide, steroid, nucleoside, nucleobase and trace element [6,7]; however, the actual quality and nutrient value of deer antler have never been well defined. Methods have been developed to identify the antler, but they are not quantitative and lack of specificity $[8,9]$. Moreover, there is no effective and accurate method to distinguish the ingredients of deer antler, as well as chemical markers for quality control, as that in Chinese Pharmacopoeia [1]. Therefore, it is important to establish a precise and sensitive method for quality evaluation of deer antler.

One of the major ingredients in deer antler is nucleoside, and indeed the nucleosides and their bases were shown to be involved in regulating and modulating various physiological processes [10,11], e.g. anti-oxidant, anti-convulsant, anti-platelet aggregation, inhibition of monoamine oxidase and anti-arrhythmic [12-14]. Furthermore, hypoxanthine, isolated from $n$-butanol extracts of $C$. nippon antler, was reported to inhibit monoamine oxidase B [15]. The qualification and quantification analyses of nucleosides and nucleobases in deer antler would be prerequisites for quality evaluation, which could be very helpful for improving its potential values. The quantitative methods have been previously developed to analyze nucleosides and nucleobases in deer antler; however, these methods focused on only a few of them [16-18]. Recently, nucleosides and nucleobases could be selected as the quality control markers in herbs and functional foods [19-21]. Here, 29 batches of deer antlers from 11 species of deer collected from different regions in China were simultaneously analyzed. An HPLC-diode array detector (DAD) method was applied to detect and quantify 10 nucleosides and nucleobases in deer antlers from different species.

\section{Materials and Methods}

\section{Chemicals and reagents}

HPLC grade acetonitrile and methanol were purchased from Merck (Darmstadt, Germany); ammonium acetate was purchased from Sigma (St. Louis, MO, USA); ultra-pure water was prepared from a Milli-Q purification system (Millipore S.A. Molsheim, France); chemical standards including uracil (1), cytidine (2), uridine (3), hypoxanthine (4), inosine (5), guanine (6), guanosine (7), thymidine (8), adenosine (9), and adenine (10) were purchased from Sigma (St. Louis, MO, USA). The purity of standard was higher than $98 \%$, determined by HPLC-DAD analysis.

\section{Deer antler materials}

The antlers of C. nippon and C. elaphus were collected from Jilin, Shanxi, Liaoning, Beijing, Xinjiang provinces and Hong Kong market

*Corresponding author: Tsim KWK, Division of Life Science, The Hong Kong University of Science and Technology, Clear Water Bay Road, Hong Kong, China, Tel: (852) 2358 7332; Fax: (852) 2358 1559; E-mail: botsim@usthk

Received February 23, 2016; Accepted April 20, 2016; Published April 27, 2016

Citation: Bi D, Zhang LML, Tang RWL, Duan R, Leung KW, et al. (2016) Quantitative Analysis of Nucleosides and Nucleobases in Deer Antler: Variation in Different Species. Pharm Anal Acta 7: 474. doi:10.4172/2153-2435.1000474

Copyright: $\odot 2016 \mathrm{Bi}$ D, et al. This is an open-access article distributed under the terms of the Creative Commons Attribution License, which permits unrestricted use, distribution, and reproduction in any medium, provided the original author and source are credited. 
in China. The antlers of Rangifer tarandus Linnaeus, C. timorensis Linnaeus, C. unicolor Kerr, C. eldi hainanus, Elaphurus davidianus Milne-Edwards, C. porcinus, C. albirostris Pre-walski, Dama dama Linnaeus, Capreolus capreolus Linnaeus were obtained and authenticated by Dalian Institute for Drug Control. The antlers of $C$. nippon and C. elaphus were authenticated by one of the authors, Dr. Tina TX Dong, according to organoleptic characteristics. All voucher specimens with number (1-29) are deposited in the Centre for Chinese Medicine at the Hong Kong University of Science and Technology.

\section{Preparation of solutions}

All samples were ground into powder (50 meshes). $0.5 \mathrm{~g}$ of dried powder was accurately weighed and extracted with $25 \mathrm{~mL}$ of $10 \%$ aqueous methanol in an ultrasonic bath $(240 \mathrm{~W})$ for $45 \mathrm{~min}$. After centrifugation $(13,000 \mathrm{rpm}, 10 \mathrm{~min})$, the supernatant was stored at 4 ${ }^{\circ} \mathrm{C}$ and filtered through a $0.45 \mu \mathrm{m}$ membrane filter before injection into the HPLC system for analysis. Stock standard solutions of individual nucleosides and nucleobases were prepared by weighing approximately $10 \mathrm{mg}$ of each compound into a volumetric flask $(10 \mathrm{~mL})$. Standards were dissolved in $10 \%$ aqueous methanol, except for guanine, which required a few drops of $2 \mathrm{M} \mathrm{HCl}$ solution for dissolution. Working standard stock solutions for calibration curves were prepared by diluting the mixed standard stock solution with $10 \%$ aqueous methanol at different concentrations, and the concentration ranges for these 10 analytes were as follows: 1, 0.61-61.08 $\mu \mathrm{g} / \mathrm{mL} ; 2,0.51-50.80 \mu \mathrm{g} / \mathrm{mL} ; 3$, $0.60-60.24 \mu \mathrm{g} / \mathrm{mL} ; \mathbf{4}, 0.75-75.00 \mu \mathrm{g} / \mathrm{mL}, 5,0.70-70.42 \mu \mathrm{g} / \mathrm{mL} ; \mathbf{6}, 0.50$ $50.00 \mu \mathrm{g} / \mathrm{mL} ; 7,0.52-51.90 \mu \mathrm{g} / \mathrm{mL} ; \mathbf{8}, 0.25-25.25 \mu \mathrm{g} / \mathrm{mL} ; 9,0.25-25.45$ $\mu \mathrm{g} / \mathrm{mL}$ and $10,0.26-25.58 \mu \mathrm{g} / \mathrm{mL}$. All solutions were stored at $4^{\circ} \mathrm{C}$ before analysis.

\section{Chromatographic conditions and instrumentation}

Analysis was performed on an Agilent 1200 series system consisting of a G1379B degasser, a G1312B binary pump, a G1367D autosampler and a G1315C DAD connected to an Agilent ChemStation running ChemStation software. Chromatographic separations were carried out on an Agilent ZORBAX SB-Aq C18 column $(5 \mu \mathrm{m}, 4.6 \times 250 \mathrm{~mm})$. The mobile phase was composed of acetonitrile (A) and $5 \mathrm{mM}$ ammonium acetate solution (B) with a gradient program elution as follows: $1 \% \mathrm{~A}$, at a flow-rate $0.4-1.0 \mathrm{~mL} / \mathrm{min}$ in $0-18 \mathrm{~min} ; 1 \% \mathrm{~A}-4 \% \mathrm{~A}$, at a flowrate $1.0 \mathrm{~mL} / \mathrm{min}$ in $18-20 \mathrm{~min} ; 4 \% \mathrm{~A}-5 \% \mathrm{~A}$, at a flow-rate $1.0 \mathrm{~mL} /$ $\min$ in $20-25 \mathrm{~min} ; 5 \% \mathrm{~A}-20 \% \mathrm{~A}$, at a flow-rate $1.0 \mathrm{~mL} / \mathrm{min}$ in $25-40$ min. Re-equilibration duration was 15 min between individual runs. Chromatographic separation was performed at room temperature. The DAD detection wavelength was set between 200 and $400 \mathrm{~nm}$, and the chromatographic peaks were measured at a wavelength of $260 \mathrm{~nm}$ to facilitate the detection of nucleosides and nucleobases. An aliquot of 10 $\mu \mathrm{L}$ was subjected for HPLC analysis.

\section{HPLC method validation}

Linear regression analysis for each analyte was performed at seven levels of concentrations, and triplicate injections were applied at each concentration. Calibration curves were constructed by plotting the peak area $(y)$ against the corresponding concentration of the standard solutions $(x, \mu \mathrm{g} / \mathrm{mL})$. The limits of detection (LODs) and quantification (LOQs) were determined at signal-to-noise ratios $(S / N)$ of about 3 and 10 , respectively. The precision of the method was evaluated by analyzing the standard solutions containing the 10 standard compounds. The intra-day variation was determined by six consecutive injections on the same day, and the inter-day variation was determined by doing duplicates on three consecutive days. Then, the relative standard deviation (RSD) of peak area for each of the marker compound was calculated, respectively. To evaluate the stability of the solution, one of the sample solutions was stored at $22^{\circ} \mathrm{C}$ and analyzed at $0,2,4,8,12$, and $24 \mathrm{~h}$, respectively. Variation was expressed as RSD. In addition, to further evaluate the repeatability of the developed assay, the same sample (\#13) was extracted and analyzed six times as described above. A recovery test was used to evaluate the accuracy of the developed method. An appropriate amount of deer antler (\#13) was weighed and spiked with known amount of each reference compound, and then extracted and analyzed as described above. Three replicates were performed for the test. The percentage recoveries were calculated as follow formula: Recovery $(\%)=100 \times$ (amount found - original amount) / amount spiked.

\section{Data analysis}

Similarity analysis was performed by professional software named Similarity Evaluation System for Chromatographic Fingerprint of Traditional Chinese Medicine (Version 2004A), which was recommended by SFDA (State Food and Drug Administration of China). The software was to employ the correlative coefficient in evaluating the similarities of different chromatograms. HCA is a multivariate analysis technique that is used to sort samples into groups. Here, different samples of deer antlers were analyzed on the contents of 10 tested analytes by using SPSS 16.0 software, and the results were subjected to HCA. PCA was performed on the contents of 10 analytes by using software of SIMCA-P.

\section{Results and Discussion}

\section{Optimization of analytical method}

In order to develop an efficient sample preparation procedure of deer antler, the variables involved in extraction, such as solvent and method, were optimized. Due to high polarity of nucleosides and nucleobases, water, $10 \%$ methanol, 30\% methanol and 50\% methanol were employed as extraction solvents, and $10 \%$ methanol was the best solvent for extraction. After comparing ultrasonic extraction with refluxing extraction, the results indicated that ultrasonic extraction was more effective than refluxing extraction in extracting nucleosides and nucleobases by using $10 \%$ methanol as extract solvent. The extraction time was also investigated, in which the sample was ultrasonically extracted for 30, 45 and $60 \mathrm{~min}$, respectively. The yield of target compounds did not increase after $45 \mathrm{~min}$. The residue after the first extraction was further extracted with $10 \%$ methanol for additional 30 min, and no nucleosides and nucleobases were detected. Therefore, the 10 analytes were completely extracted within $45 \mathrm{~min}$, and ultrasonic extraction with $10 \%$ aqueous methanol for $45 \mathrm{~min}$ in a single step was selected for routine usage.

In order to obtain good separation and shorten analysis time of the experiment, the chromatographic conditions including the column, compositions of mobile phase, gradient elution program and flow rate were investigated and optimized. In our preliminary tests, different reversed-phase columns such as Zorbax Ecliopse XDB C18, Luna C18, Zorbax SB-Aq C18, Inertsil ODS-4 C18, and Alltima C18 were investigated. The results showed that the nucleosides and nucleobases were the compounds with high polarity, which were easily separated on Zorbax SB-Aq C18 column. For the mobile phase, acetonitrile performed better as an organic modifier than methanol and was thus 
chosen to constitute the mobile phase with water and $5 \mathrm{mM}$ ammonium acetate aqueous solution, it was found that $5 \mathrm{mM}$ ammonium acetate aqueous solution could achieve the ideal separation. According to the UV absorption maxima of 10 reference compounds on UV spectra obtained by HPLC-DAD, the wavelength was set at $260 \mathrm{~nm}$ that provided an optimum $S / N$ for simultaneous quantitative analysis of the 10 nucleosides and nucleobases (Figure 1). The HPLC chromatograms of standard analytes and deer antler samples were shown in Figure 2. Identification of investigated compounds was achieved by comparison of their retention times and their UV spectra with those of reference compounds under the same condition or by spiking the samples with stock standard solutions.

The calibration curves were constructed by plotting the peak area (y) against the corresponding concentration of the standard solutions $(x, \mu \mathrm{g} / \mathrm{mL})$. The calculated results were given in Supplementary Table 1. The good linearity (correlation coefficient value $r^{2} \geq 0.9995$ ) was achieved in relatively wide concentration ranges for all the analytes. For 10 analytes, the LODs ranged from 0.031 to $0.141 \mu \mathrm{g} / \mathrm{mL}$, and LOQs ranged from 0.097 to $0.423 \mu \mathrm{g} / \mathrm{mL}$. The overall intra- and interday variations were less than $1.77 \%$ for the 10 analytes. Validation studies of this method showed a good repeatability with RSD less than $4.67 \%(n=6)$ for investigated compounds (Supplementary Table 2). As shown in Supplementary Table 3, the established method had an excellent accuracy with an overall recovery from $95.00 \%$ to $101.92 \%$ $(n=3)$ for the analytes. Therefore, the developed HPLC-DAD method was precise, accurate and sensitive enough for the simultaneous quantitative determination of the 10 nucleosides and nucleobases in deer antler.

\section{Nucleosides and nucleobases in deer antler}

The established HPLC-DAD method was applied to simultaneous quantification of the 10 nucleosides and nucleobases in 29 batches of deer antlers from 11 species, including C. nippon, C. elaphus, C. timorensis, C. unicolor, C. eldi hainanus, C. porcinus, C. albirostris, $R$. tarandus, E. davidianus, D. dama and C. capreolus. The sample code and their places of collection were listed in Table 1 . These antlers were extracted according to the established protocol as above. Thereafter, HPLC fingerprints were generated for the 29 samples (Figure 2). The fingerprint similarity of those antlers was calibrated, and C. nippon and C. elaphus showed the greatest value (Table 1 ). The worst similarity was revealed in E. davidianus. In addition, the contents of total nucleosides

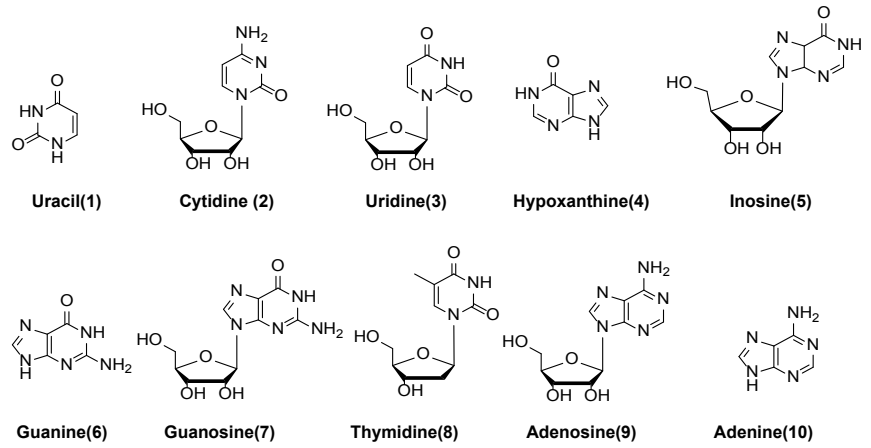

The chemical structures of 10 marker chemicals, including uracil (1), cytidine (2), uridine (3), hypoxanthine (4), inosine (5), guanine (6), guanosine (7), thymidine (8), adenosine (9), and adenine (10), were shown. The notions were in accordance with the HPLC chromatograms in Figure 2.

Figure 1: Chemical structures of the 10 nucleosides and nucleobases analyzed in deer antler.

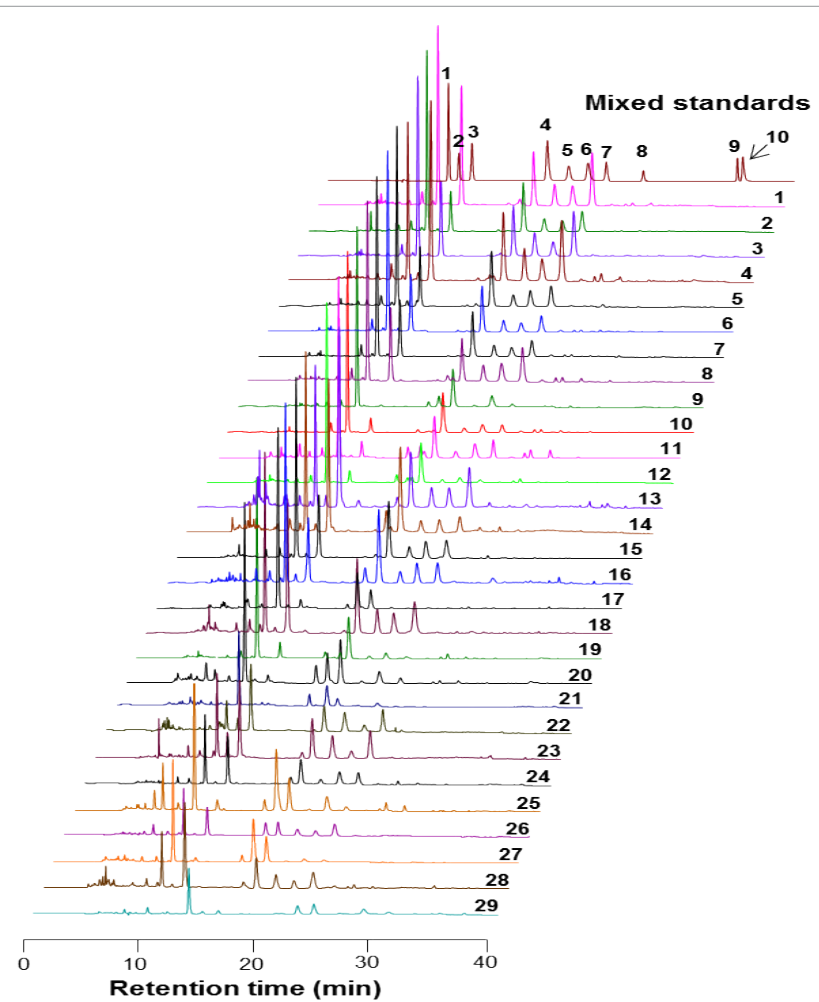

HPLC chromatograms of 29 samples (number 1 to 29 for different samples, shown in Table 1) at $260 \mathrm{~nm}$ were shown, $n=5$. The first profile showed the migration of marker chemicals, i.e. uracil (1), cytidine (2), uridine (3) hypoxanthine (4), inosine (5), guanine (6), guanosine (7), thymidine (8), adenosine (9), and adenine (10).

Figure 2: The representative HPLC chromatograms of mixed standards and 11 deer species.

and nucleobases in antlers were determined. The contents of nucleosides and nucleobases varied significantly in different species, as well as their habitats: the total content was ranged from 233.61 to $3,089.93 \mu \mathrm{g} / \mathrm{g}$ (Table 2). In general, these antlers contained high amounts of uracil, uridine, hypoxanthine, inosine and guanosine. In contrast, adenosine and adenine were hardly to be detected in the 29 samples. The contents of both total and individual nucleosides and nucleobases in C. nippon and C. elaphus varied greatly from different habitats (Table 2), which could be accounted for varying climate, local environment and feeding [22]. The contents of 10 tested analytes in 29 antler samples were input to HCA and PCA analyses. Both HCA and PCA could not discriminate deer antlers from different species (Figure 3).

To distinguish Cervi Cornu Pantotrichum from its counterfeits, HPLC fingerprint focusing on the amounts of nucleosides and nucleobases has been developed. Taking nucleosides and nucleobases as the only parameters for identification could not be possible: because these chemicals vary a lot in different collection areas as well as species origins. Nevertheless, the HPLC fingerprints of C. nippon and C. elaphus antlers shared a close similarity, regardless where they were collected, suggesting a possible application of this method.

Proteins have been proposed for identification purpose. From the extracts of deer antlers, specific protein bands were identified by SDSPAGE $[23,24]$. Moreover, 5 proteins were identified from deer antler, e.g. $\beta$-3 subtype of hemoglobin, antimicrobial peptide, peptidoglycan recognition protein, $\beta$-c subtype of hemoglobin and pre-pro serum 
Citation: Bi D, Zhang LML, Tang RWL, Duan R, Leung KW, et al. (2016) Quantitative Analysis of Nucleosides and Nucleobases in Deer Antler: Variation in Different Species. Pharm Anal Acta 7: 474. doi:10.4172/2153-2435.1000474

Page 4 of 6
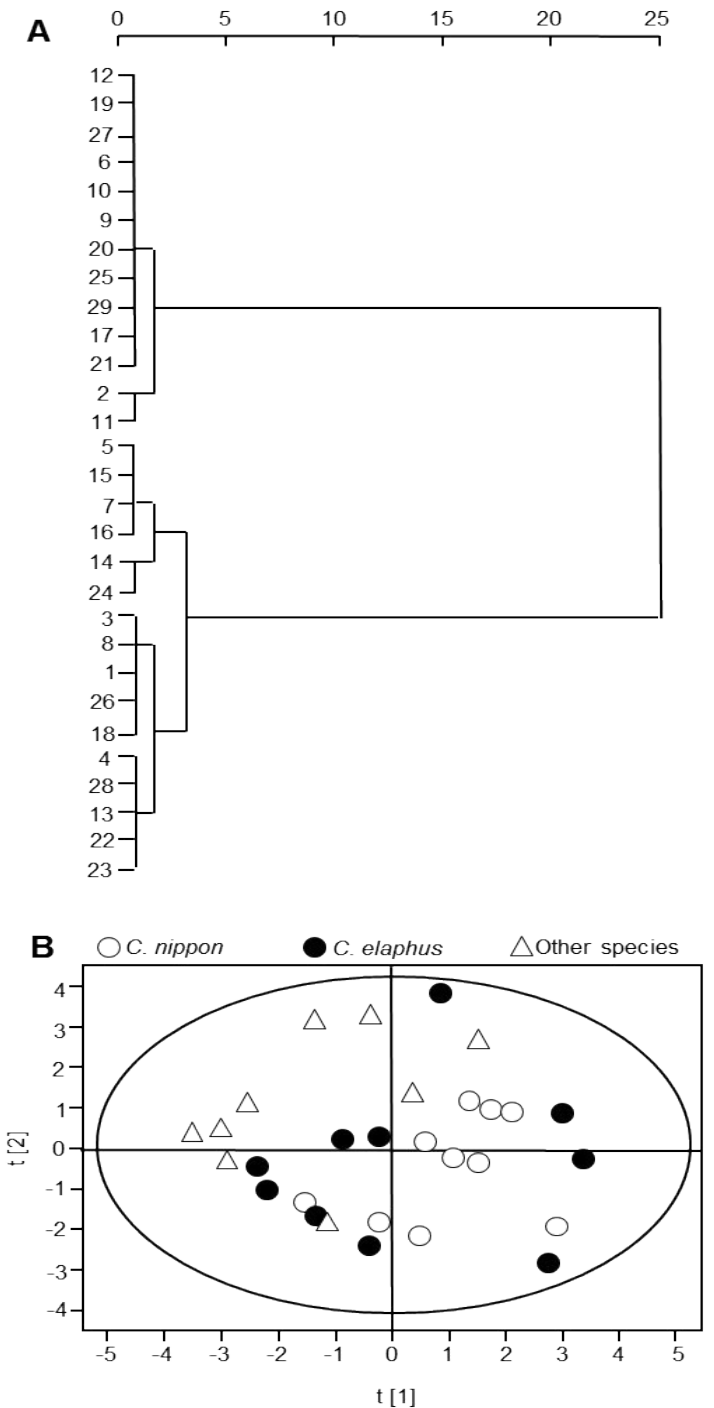

(A): Dendrograms of hierarchical cluster analysis for the 29 samples of deer antlers. The loading plot was performed with the amounts of uracil, cytidine, uridine, hypoxanthine, inosine, guanine, guanosine, thymidine, adenosine, and adenine as shown in Table 2.

(B): Hierarchical clustering analysis for the 29 samples. The input data was as in (A).

Figure 3: Analysis of 29 samples of deer antlers according to their content of nucleosides and nucleobases.

albumin [25]. Hormones have been identified in deer antlers, e.g. estradiol, testosterone, insulin-like growth factor-1 [26]. Nevertheless, the identified chemicals so far are not indicative for authentication of Cervi Cornu Pantotrichum from its counterfeits. Besides chemical analysis, DNA method has been used in authenticating Cervi Cornu Pantotrichum from its counterfeit antlers, which includes the sequencing of mitochondrial cytochrome $b$ and cytochrome $c$ oxidase subunit 1 genes [27], and PCR-RFLP analysis by using a set of primers flanking a conserved region of the mitochondrial $12 \mathrm{~S}$ rRNA gene [28]. However, the molecular identification is not able to identify deer antlers of different quality. In addition, the employment of electronic nose has been reported in identifying fake antlers from Cervi Cornu Pantotrichum [29].

In conclusion, this is the first report using a simultaneous determination of 10 nucleosides and nucleobases in deer antlers from 11 species with HPLC-DAD method, which has been shown here to be a simple, rapid and accurate approach. The present results showed that most of deer antlers were rich in the content of nucleosides and nucleobases. The derived results showed similarity value in chemical fingerprints of C. nippon and C. elaphus was the highest. However, the investigated analytes could not fully discriminate deer antlers from different species. Therefore, specific chemical markers for quality control to discriminate deer antlers from different species would be studied in subsequent work.

\section{Acknowledgements}

Supported by Hong Kong Research Grants Council Theme-based Research Scheme (T13-607/12R), ITF (UIM/254), GRF (663012, 662713, M-HKUST604/13), TUYF15SC01, The Hong Kong Jockey Club Charities Trust (HKJCCT12SC01) and Foundation of The Awareness of Nature (TAON12SC01) to Karl Tsim.

\begin{tabular}{|c|c|c|c|}
\hline Sample & Species & Sources & Similarity ${ }^{a, b}$ \\
\hline 1 & Cervus nippon & Hongkong market & $0.968^{a}$ \\
\hline 2 & Cervus nippon & Hongkong market & $0.994^{a}$ \\
\hline 3 & Cervus nippon & Hongkong market & $0.971^{a}$ \\
\hline 4 & Cervus nippon & Hongkong market & $0.836^{a}$ \\
\hline 5 & Cervus nippon & Shanxi & $0.997^{a}$ \\
\hline 6 & Cervus nippon & Liaoning & $0.969^{a}$ \\
\hline 7 & Cervus nippon & Liaoning & $0.998^{a}$ \\
\hline 8 & Cervus nippon & Beijing & $0.979^{a}$ \\
\hline 9 & Cervus nippon & Jilin & $0.936^{a}$ \\
\hline 10 & Cervus nippon & Jilin & $0.969^{a}$ \\
\hline 11 & Cervus elaphus & Hongkong market & $0.962^{a}$ \\
\hline 12 & Cervus elaphus & Hongkong market & $0.965^{a}$ \\
\hline 13 & Cervus elaphus & Hongkong market & $0.779^{a}$ \\
\hline 14 & Cervus elaphus & Hongkong market & $0.918^{a}$ \\
\hline 15 & Cervus elaphus & Liaoning & $0.986^{a}$ \\
\hline 16 & Cervus elaphus & Jilin & $0.974^{a}$ \\
\hline 17 & Cervus elaphus & Xinjiang & $0.887^{a}$ \\
\hline 18 & Cervus elaphus & Jilin & $0.909^{a}$ \\
\hline 19 & Cervus elaphus & Jilin & $0.965^{a}$ \\
\hline 20 & Cervus elaphus & Jilin & $0.927^{a}$ \\
\hline 21 & Rangifer tarandus & Jilin & $0.112^{b}$ \\
\hline 22 & Cervus timorensis & Beijing & $0.502^{b}$ \\
\hline 23 & Cervus unicolor & Sichuan & $0.548^{b}$ \\
\hline 24 & Cervus eldi hainanus & Hainan & $0.817^{\mathrm{b}}$ \\
\hline 25 & Elaphurus davidianus & Liaoning & $0.852^{b}$ \\
\hline 26 & Cervus porcinus & Beijing & $0.749^{b}$ \\
\hline 27 & Cervus albirostris & Sichuan & $0.814^{b}$ \\
\hline 28 & Dada dama & New Zealand & $0.604^{b}$ \\
\hline 29 & Capreolus capreolus & Jilin & $0.935^{b}$ \\
\hline
\end{tabular}

a Similarities of each chromatogram to the corresponding representative standard fingerprint of each species by Similarity Evaluation System, $n=4$.

b Similarities of each species to the corresponding representative standard fingerprint of $C$. nippon by Similarity Evaluation system, $n=4$

Table 1: Similarities of HPLC fingerprints deriving from different species of deer antlers. 
Citation: Bi D, Zhang LML, Tang RWL, Duan R, Leung KW, et al. (2016) Quantitative Analysis of Nucleosides and Nucleobases in Deer Antler: Variation in Different Species. Pharm Anal Acta 7: 474. doi:10.4172/2153-2435.1000474

Page 5 of 6

\begin{tabular}{|c|c|c|c|c|c|c|c|c|c|c|c|}
\hline Sample & Uracil & Cytidine & Uridine & Hypoxanthine & Inosine & Guanine & Guanosine & Thymidine & Adenosine & Adenine & Total \\
\hline 1 & 409.08 & 10.32 & 706.43 & 331.7 & 362.74 & 201.75 & 617.12 & 30.22 & $\mathrm{nd}^{\mathrm{a}}$ & nd & 2669.36 \\
\hline 2 & 720.61 & 23.58 & 385.66 & 554.86 & 401.68 & 196.6 & 454.87 & 170.9 & nd & nd & 2755.76 \\
\hline 3 & 449.04 & 19.19 & 496.09 & 343.13 & 432.74 & 159.28 & 556.63 & nd & $\operatorname{tr}^{b}$ & nd & 2456.1 \\
\hline 4 & 230.36 & 36.93 & 711.29 & 289.1 & 356.11 & 142.13 & 471.32 & 39.81 & $\operatorname{tr}$ & nd & 2277.05 \\
\hline 5 & 542.64 & 47.96 & 422.72 & 430.34 & 240.75 & 188.17 & 317.54 & 21.88 & $\operatorname{tr}$ & nd & 2212 \\
\hline 6 & 770.77 & nd & 168.02 & 471.61 & 150.96 & 104.25 & 165.2 & 60.21 & $\mathrm{Nd}$ & nd & 1891.02 \\
\hline 7 & 688.15 & 26.59 & 566.92 & 478.42 & 332.34 & 156.59 & 335.5 & 26.88 & $\operatorname{tr}$ & nd & 2611.39 \\
\hline 8 & 405.03 & 1.95 & 443.41 & 270.25 & 277.85 & 191.61 & 414.77 & 31.33 & $\operatorname{tr}$ & nd & 2036.2 \\
\hline 9 & 589.34 & nd & 5.38 & 343.82 & 22.97 & 167.79 & 51.94 & 8.45 & $\operatorname{tr}$ & nd & 1189.69 \\
\hline 10 & 888.05 & 6.87 & 192.65 & 549.92 & 163.64 & 166.32 & 183.35 & 47.87 & $\operatorname{tr}$ & nd & 2198.67 \\
\hline 11 & 907.84 & 25.44 & 196.33 & 587.92 & 241.54 & 305.42 & 393.22 & 148.2 & nd & nd & 2805.91 \\
\hline 12 & 668.3 & nd & 109.5 & 405.94 & 72.29 & 92 & 62.83 & 59.34 & nd & nd & 1470.2 \\
\hline 13 & 231.11 & 48.15 & 807.86 & 251.53 & 247.79 & 154.12 & 419.41 & 13.31 & 13.09 & 10.57 & 2196.94 \\
\hline 14 & 256.67 & 24.38 & 536.97 & 330.66 & 110.66 & 67.36 & 111.55 & 24.17 & $\operatorname{tr}$ & $\operatorname{tr}$ & 1462.42 \\
\hline 15 & 688.05 & 54.05 & 658.42 & 604.03 & 311.79 & 277.66 & 465.01 & 30.92 & nd & nd & 3089.93 \\
\hline 16 & 340.63 & 48.18 & 375.37 & 377.44 & 156.74 & 154.1 & 220.17 & nd & $\operatorname{tr}$ & $\operatorname{tr}$ & 1672.63 \\
\hline 17 & 847.27 & 36.69 & 86.34 & 234.14 & 35.89 & nd & nd & 22.31 & $\operatorname{tr}$ & nd & 1265.64 \\
\hline 18 & 464.03 & 41.03 & 860.09 & 507.93 & 429.74 & 212.23 & 529.83 & 10.94 & $\operatorname{tr}$ & $\operatorname{tr}$ & 3055.82 \\
\hline 19 & 859.98 & nd & 176.96 & 521.12 & 89.49 & 101.42 & 59.25 & 82.68 & $\operatorname{tr}$ & $\operatorname{tr}$ & 1890.9 \\
\hline 20 & 326.2 & 10.68 & 24.27 & 199.7 & 16.36 & 89.43 & 47.82 & 16.39 & $\operatorname{tr}$ & $\operatorname{tr}$ & 730.85 \\
\hline 21 & 150.93 & nd & 19.05 & 39.5 & $\operatorname{tr}$ & 24.13 & $\operatorname{Tr}$ & nd & $\operatorname{tr}$ & $\operatorname{tr}$ & 233.61 \\
\hline 22 & 77.49 & 90.55 & 447.36 & 189.22 & 371.27 & 70.82 & 272.69 & nd & $\operatorname{tr}$ & 6.82 & 1526.22 \\
\hline 23 & 218.37 & 177.74 & 539.75 & 310.72 & 447.41 & 85.49 & 375.8 & nd & $\operatorname{tr}$ & nd & 2155.28 \\
\hline 24 & 176.58 & 17.74 & 319.26 & 188.69 & 86.87 & 137.93 & 163.66 & 29.33 & 17.71 & 9.89 & 1147.66 \\
\hline 25 & 622.22 & nd & 90.7 & 265.72 & 21.14 & 177.44 & 70.88 & 87.62 & nd & nd & 1335.72 \\
\hline 26 & 102.39 & 9.59 & 153.5 & 76.09 & 95.4 & 41.07 & 136.98 & nd & nd & nd & 615.02 \\
\hline 27 & 273.95 & 7.86 & 32.62 & 186.98 & 16.55 & 28.14 & 42.82 & nd & nd & nd & 588.92 \\
\hline 28 & 172.84 & 25.4 & 671.15 & 257.59 & 318.5 & 100.16 & 311.79 & 32.89 & nd & nd & 1890.32 \\
\hline 29 & 115.39 & 15.9 & 22.74 & 70.07 & 14.77 & 60.27 & 30.8 & 14.62 & nd & nd & 344.56 \\
\hline
\end{tabular}

The data in $\mu \mathrm{g} / \mathrm{g}$ of dried antler, $n=3$ to 4

and: not detected

${ }^{\mathrm{b}}$ tr: below the limit of quantification

Table 2: Contents of nucleosides and nucleobases in deer antlers

\section{References}

1. Editorial Committee of China Pharmacopoeia (2015) Pharmacopoeia of the People's Republic of China. China Medical Science Press, Beijing 1: 323.

2. Yan Z, Yuan RY, Wan CY, Zhang ZH, Cai LP, et al. (2009) Study on fingerprint of Corn Cervi Pantotrichum by HPCE. Food Sci Technol 34: 254-258.

3. Tsujibo H, Miyake $\mathrm{Y}$, Maruyama K, Inamori Y (1987) Hypotensive compounds isolated from alcohol extract of the un-ossified horn of Cervus elaphus L. var xanthopygus Milne-Edwarg (Rokujo) I Isolation of lysophosphatidyl choline as a hypotensive principle and structure - activity study of related compounds. Chem Pharm Bull 35: 654-659.

4. Wang BX, Zhao XH, Qi SB, Yang XW, Kaneko S, et al. (1988) Stimulating effect of deer antler extract on protein synthesis in senescence-accelerated mice in vivo. Chem Pharm Bull (Tokyo) 36: 2593-2598.

5. Kang SK, Kim KS, Kim SI, Chung KH, Lee IS, et al. (2006) Immunosuppressive activity of deer antler extracts of Cervus korean TEMMINCK var. mantchuricus Swinhoe, on type II collagen-induced arthritis. In Vitro Cell Dev Biol Anim 42 100-107.

6. Qiu FP, Du HX, Wang ZB (2009) Purification of pilose antler polysaccharides. $J$ Harbin Inst Technol 41: 223-225.

7. Li HP (2003) The velvet chemical compositions of velvet - deer breeds or strains in China. J NE Forestry Uni 31: 26-28.

8. Yang H, Shen YP, Xu Y, Maqueda AS, Zheng J, et al. (2015) A novel strategy for the discrimination of gelatinous Chinese medicines based on enzymatic digestion followed by nano-flow liquid chromatography in tandem with orbitrap mass spectrum detection. Int J Nanomedicine 10: 4947-4955.

9. Zhang C, Su J (2014) Application of near infrared spectroscopy to the analysis and fast quality assessment of traditional Chinese medicinal products. Acta Pharm Sin B 4: 182-192.
10. Ralevic V, Burnstock G (1998) Receptors for purines and pyrimidines. Pharmacol Rev 50: 413-492.

11. Jacobson KA, Jarvis MF, Williams M (2002) Purine and pyrimidine (P2) receptors as drug targets. J Med Chem 45: 4057-4093.

12. Schmidt AP, Lara DR, de Faria Maraschin J, da Silveira Perla A, Onofre Souza D (2000) Guanosine and GMP prevent seizures induced by quinolinic acid in mice. Brain Res 864: 40-43.

13. VirÃ $i g ~ L, S z a b \tilde{A}^{3}$ C (2001) Purines inhibit poly(ADP-ribose) polymerase activation and modulate oxidant-induced cell death. FASEB J 15: 99-107.

14. Anfossi G, Russo I, Massucco P, Mattiello L, Cavalot F, et al. (2002) Adenosine increases human platelet levels of 30,50-cGMP through nitric oxide: possible role in its anti-aggregating effect. Thromb Res 105: 71-78.

15. Wang BX, Zhao XH, Yang XW, Kaneko S, Hattori M, et al. (1988) Identification of the inhibitor for monoamine oxidase $B$ in the extract from deer antler (Rokujo) J Med Pharm Soc Wakan-Yaku 5: 116-122.

16. Yang XW (1995) Quantitative HPLC analyses of nucleosides and nucleobases and inhibition of MAO activities in sika deer pilose antler and red deer pilose antler. Chin Tradit Herb Drugs 26: 17-19.

17. Zhou R, Li SF (2009) RP - HPLC simultaneous determination of three biological base in antler velvet. Chin J Pharm Anal 29: 575-578.

18. Zong Y, Wang Y, Li H, Li N, Zhang H, et al. (2014) Simultaneous quantification and splenocyte-proliferating activities of nucleosides and bases in Cervi Cornu Pantotrichum. Pharmacogn Mag 10: 391-397.

19. Cao XW, Li J, Chen SB, Li XB, Xiao PG, et al. (2010) Simultaneous determination of nine nucleosides and nucleobases in different Fritillaria species by HPLCdiode array detector. J Sep Sci 33: 1587-1594. 
Citation: Bi D, Zhang LML, Tang RWL, Duan R, Leung KW, et al. (2016) Quantitative Analysis of Nucleosides and Nucleobases in Deer Antler: Variation in Different Species. Pharm Anal Acta 7: 474. doi:10.4172/2153-2435.1000474

20. Guo S, Duan JA, Tang YP, Zhu ZH, Qian YF, et al. (2010) Characterization of nucleosides and nucleobases in fruits of Ziziphus jujuba by UPLC-DAD-MS. $J$ Agric Food Chem 58: 10774-10780.

21. Ranogajec A, Beluhan S, Smit Z (2010) Analysis of nucleosides and monophosphate nucleotides from mushrooms with reversed-phase HPLC. J Sep Sci 33: 1024-1033.

22. Wen H, Jeon B, Moon S, Song Y, Kang S, et al. (2010) Differentiation of antlers from deer on different feeds using an NMR-based metabolomics approach. Arch Pharm Res 33: 1227-1234.

23. Su FY, Li HP, Wang YM, Huang YX, Xiao SM, et al. (2001) Protein component extraction and its bioactivity determination of sika deer antler base. Animal Science and Veterinary Medicine 18: 18-20

24. Tang RN, Zhao Y, Sun XD, Qu XB (2008) Comparison of water soluble total protein in velvet, antler plate and bone of sika deer. Jilin Journal of Traditional Chinese Medicine 28: 295-29.
25. Huang $P$, Zhao $Y$ Niu F Tang RN, LiYQ et al. (2010) Isolation and identification of sika antler base soluble proteins. Asia-Pacific Traditional Medicine 6: 28-30.

26. Tseng SH, Sung CH, Chen LG, Lai YJ, Chang WS, et al. (2014) Comparison of chemical compositions and osteoprotective effects of different sections of velvet antler. J Ethnopharmacol 151: 352-360.

27. Gao L, Xia W, Ai J, Li M, Yuan G, et al. (2015) Development of multiplex PCR assay for authentication of Cornu Cervi Pantotrichum in traditional Chinese medicine based on cytochrome $b$ and $C$ oxidase subunit 1 genes. Mitochondrial DNA .

28. Fajardo V, GonzÃ $\tilde{i}_{\text {lez I, LÃ }}^{3}$ pez-Calleja I, MartÃn I, Herñ̃ $n d e z$ PE, et al (2006) PCR-RFLP authentication of meats from red deer (Cervus elaphus), fallow deer (Dama dama), roe deer (Capreolus capreolus), cattle (Bos taurus), sheep (Ovis aries), and goat (Capra hircus). J Agric Food Chem 54: 1144-1150.

29. Xu GJ, Liu CS, Liao CL, Ren XL, Zhang XY, et al. (2016) Rapid and accurate identification of adulterants via an electronic nose and DNA identification platform: identification of fake velvet antlers as an example. Journal of Sensors 2016: ahead of print 